OPEN ACCESS

Edited by:

Dhaval Vyas,

The University of Queensland,

Australia

Reviewed by:

Amra Delic,

University of Sarajevo, Bosnia and

Herzegovina

Mary Uhl-Bien,

Texas Christian University,

United States

*Correspondence:

Martin C. Nwadiugwu

mnwadiugwu@unomaha.edu

Specialty section:

This article was submitted to

Human-Media Interaction,

a section of the journal

Frontiers in Computer Science

Received: 11 July 2020 Accepted: 15 February 2021

Published: 29 March 2021

Citation:

Nwadiugwu MC and Nwadiugwu CC

(2021) Influencing Followership:

Understanding the Perspective of

Those Leading Active Discussions

on Quora.

Front. Comput. Sci. 3:582242.

doi: 10.3389/fcomp.2021.582242

\section{Influencing Followership:} Understanding the Perspective of Those Leading Active Discussions on Quora

\author{
Martin C. Nwadiugwu ${ }^{1 *}$ and Cynthia C. Nwadiugwu ${ }^{2}$ \\ ${ }^{1}$ Department of Biomedical Informatics, College of Information Science and Technology (IS\&T), University of Nebraska Omaha, \\ Omaha, NE, United States, ${ }^{2}$ Fenwick Healthcare, Wigan, United Kingdom
}

As social media influence become increasingly popular, understanding why some posts are highly followed than others, especially from the perspective of those leading the discussion allows us to gain insight on how followership is being influenced. A qualitative study of eight participants leading active discussions on Quora was conducted using semistructured in-depth interviews, followed by thematic analysis. The open coding method was used to iteratively code related answers to develop themes. Results suggest that copyright tactics, controversial answers and sharing new information are some of the mechanisms for influencing followership. These mechanisms are built overtime through conscious strong engagement and by writing a consistently well-thought-out answer. The motivation for leading and writing answers on Quora were more intrinsic than extrinsic, and most participants believed influencing followership should not be a concern if one has the right message.

Keywords: followership, influence, leadership, online communities, Quora

\section{INTRODUCTION}

Quora is a social media online platform that brings people with diverse perspectives to understand each other in a culture of knowledge sharing (Quora, 2020). It is a popular question and answer site that differs from others because it integrates social network into its basic structure (Wang et al., 2013; Maity et al., 2017). On the platform, the best answers are seemingly identified by upvotes and downvotes (Patil and Lee, 2016). It has been previously reported that Quora draws about 200 million visitors monthly, and as at 2017, over 13 million questions have been asked (D'Angelo, 2017; Quora, 2017; Peh, 2017). Interestingly, while there are many questions and answers on the platform, it is not widely known why some answers are more influential than others (Paul et al., 2012). It is pertinent to be thoroughly familiar with the experiences and perspectives of those writing answers and leading discussions on Quora to comprehend how followership is being influenced.

Assessment of Quora shows it follows the principles of online community design suggested by Kim (2003). The platform has a defined purpose that understand the intended users and the services it intends to deliver in the "Why Quora Exist" section of the home page that also highlights the mission statement of the community which is: "to share and grow the world's knowledge" (Quora, 2020). An overview of the website content is shown in a simple and usable way allowing visitors to navigate the design of member profiles that evolve over time, and to be made aware of their rights 
and responsibilities in the community which can be seen by clicking the "conduct policy" link at the bottom of the home page. While there is a clear code of conduct, controversy, debate and allowing members to be different in a way that is not disruptive is permitted and this creates the fun (Kim, 2003). Ranking of members and their level of completed membership profile is also visible to the user so that more accurate information can be updated. As a Q\&A online platform, discussion areas exist, engaging the community with effective leadership (Kim, 2003). New members are allowed to lead discussions and answer questions in agreement with the principle of creating a balance that accommodates new members without alienating those that are active (Kim, 2003).

The focus of this study is on effective leadership within discussion areas on Quora. It aims to gain insight into understanding why some answers are highly followed; specifically, to understand if those leading active discussions are self-aware and if they play a role in influencing followership, comments and upvotes. The social mechanisms and decision-making processes that help them judge if an answer would be trending is a focus of this study. For example, 1) What do social media influencers hope to gain from writing answers or leading a discussion? 2) What influences active discussion on Quora? In other words, which scenarios will encourage a post to trend and 3) how does the topic affect user experience and followership on Quora?

A closer observation of the platform shows that each user has a profile history that keeps track of all answers and questions an individual had posted, the number of shares and following they have. The Technology space within Quora (observed from February 6 to March 15, 2020) was selected for this study, and the activities of people who had posted answers that received considerable activity, that is, posts that have garnered about 50 or more comments, upvotes and shares were observed. While some users on the platform could be identified with pseudonyms, only those who used supposedly real identities were further observed and selected as potential participants, because real identities build perception of trust for high quality answers (Kollock, 1998; Paul et al., 2012). Although popularity based on real identities may be biased due to the fact that users may in general have an attachment and preference to some notoriety, which can influence the voting and promotion of answers that may not be novel (Barabási and Albert, 1999); the social voting is still considered as a way of identifying quality answers (Paul et al., 2012) and was used in this study as the criteria for selecting participants leading active discussions on Quora.

The remaining sections of the study will highlight the purpose and methodology used. Next, a weaving of the in-depth interview from the participants will be synthesized to answer the study question of how followership is being influenced. Answers to questions about the participant's understanding of leadership, motives for leading online discussions, self-awareness of user behavior, mechanisms for influencing followership and how unrelated questions affect user experience will be sought. The study will conclude with a summary of the narration and how members of online communities may benefit from future design improvements.

\section{Purpose of the Study}

Although every member in an online community can exhibit leadership behavior, some are more effective in influencing others (Zhu et al., 2012). According to Weeks et al. (2017) opinion leaders and highly active users in online communities can be influential in persuading their peers and believe they are both indirectly and directly able to persuade others. In a study on the effectiveness of leadership in online communities, Zhu et al. (2012) found that person-focused leaders are more effective in influencing others to contribute, while legitimate leaders are the more influential. However, leadership can only exist when there is a followership, and the advent of the internet has given rise to global followership allowing leaders and their following to be united in a partnering process (Tolstikov-Mast and Yulia, 2016). It is therefore important to know the mechanisms that influence followership via the internet and why some answers and discussion posts in online communities have more participation than others, especially given the theory that leadership is founded on the desire for control over others (Ford and Harding, 2018).

\section{METHODOLOGY}

The study was conducted using a semi-structured, in-depth interview. Eight participants were selected for the study comprising of six males and two females as seen in Table 1 using non-probability sampling (Quota sampling), which selects participants according to some particular characteristics (Evans and Rooney, 2013). Only people who are members of the Technology space within Quora and who posted answers with 50 or more comments, upvotes and shares were sampled. While quota sampling may influence the external validity of a study, it is a valid method when the goal is not to describe a population (Evans and Rooney, 2013). This sampling technique was used since the primary goal of the study was to understand mechanisms influencing followership and not to describe the population of interest. All participants were above 18 years of age. A pilot test was carried out to ensure adequate refinement of the interview questions according to the interview guide (Creswell, 2007). The interviews were conducted via Zoom and were audiorecorded except for two that were text-based. The interviews lasted for about $30 \mathrm{~min}$.

The terms of confidentiality were addressed and the participants gave recorded consent to use the transcript of the interview in the study. Participant names were de-identified as seen in Table $\mathbf{1}$ in line with suggestions that pseudonyms, and disguising person names are recommended for protecting confidentiality of research subjects (Bruckman, 2006). The participants were sent the initial transcript at the end of the interview to allow them make clarifications on possible errors in the transcription process. The following open-ended questions were asked: 
TABLE 1 | Characteristics of selected participant.

\begin{tabular}{|c|c|c|c|c|c|c|}
\hline Participants & Gender & Country & Following & Answers & Content views & Job highlight \\
\hline P01 & Male & United States & 245 & 129 & 932,400 & College sudent \\
\hline P02 & Male & United States & 510 & 120 & 807,300 & Online writer \\
\hline P02 & Male & Nigeria & 504 & 3,923 & $7,600,000$ & Blogger \\
\hline P04 & Female & United States & 4,310 & 2,787 & $5,600,000$ & Patreon analyst \\
\hline P05 & Male & United States & 1,075 & 4,436 & $13,300,000$ & Business adviser/writer \\
\hline P06 & Male & France & 74,532 & 387 & $29,600,000$ & Stand-up comedian \\
\hline P07 & Male & United States & 6,558 & 566 & $7,100,000$ & College student \\
\hline P08 & Female & United States & 3,828 & 319 & $2,900,000$ & Borderline advocate \\
\hline
\end{tabular}

TABLE 2 | Saturation Assessment.

\begin{tabular}{|c|c|c|c|c|c|c|c|c|}
\hline \multirow[t]{2}{*}{ Participant no. } & \multicolumn{4}{|c|}{ Base size } & \multicolumn{2}{|c|}{$\begin{array}{l}\text { Run } \\
\text { length }\end{array}$} & \multicolumn{2}{|c|}{$\begin{array}{l}\text { Run } \\
\text { length }\end{array}$} \\
\hline & 1 & 2 & 3 & 4 & 5 & 6 & 7 & 8 \\
\hline $\begin{array}{l}\text { New themes per } \\
\text { individual interview }\end{array}$ & 3 & 0 & 1 & 0 & 1 & 0 & 0 & 0 \\
\hline New themes in run & & & & 4 & & $\begin{array}{c}1 \\
25 \%\end{array}$ & & $\begin{array}{c}0 \\
0 \%\end{array}$ \\
\hline
\end{tabular}

1. Tell me about your understanding of being a leader on Quora? 2. What do you hope to gain from leading a discussion on Quora?

3. Tell me about any concern you have in leading an online discussion?

4. What scenarios will make a post trending on Quora?

5. Do people look up to suggestions on Quora in making their decisions?

6. How do unrelated questions affect user experience/behavior? 7. Anything else you would like to share?

At the end of the interview and transcription process, the data were analyzed using a thematic methodology that incorporated an inductive approach to generate explanations from the data [Warwick Institute for Employment Research (IER), 2014]. The rationale for using this method was because the aim of the interview follows a strategy that seeks an explanation of mechanisms on how to gain followership and the thematic analysis provides a well-structured approach in explaining the data (Nowell et al., 2017).

The thematic saturation was calculated using the method suggested by Guest et al. (2020) where incoming data is weighed against the information that exist and if it is $\leq 5 \%$ information threshold, saturation is reached. This is shown in Table 2 where a base size of themes from the first four interviews and a subsequent run length of two is used in the calculations. The run length is divided by the base size; the run length is the numerator while the base size is the denominator. From Table 2, the resulting sum of the base size is four and a run length of two (additional themes from the next two interviews) gives $25 \%$ change over base size. Since this is not $\leq 5 \%$ information threshold, the base length is again divided by the sum of the number of themes from the next run length which is 0 . The output of the calculation is 0 which is $\leq 5 \%$ threshold, therefore, saturation is deemed to be achieved.

\section{Participant Interview}

Table 3 shows the thematic analytic approach used to code the interview transcripts and to iteratively develop themes related to the interview questions as suggested by Strauss and Corbin (1990), and Vollstedt and Rezat (2019).

\section{Thematic Analysis}

\section{Leadership and Followership Relationship}

Studies have posited that the experience of leadership varies in relation to how their following differ in acting out their roles (Carsten et al., 2018). Followership role orientation co-produces leadership outcome of influence, motivation, and perceived follower support (Carsten et al., 2018). In other words, influential leadership is hinged on the perception of support from fans which motivates the online leader. The ability to influence participation on Quora was highlighted by seven participants as a feature of leadership. One participant said: "I would agree and categorize being a leader as one who has lots of followership. I also think being a leader is being one with integrity and that's what is kind of falls down to"-P01. Apart from having a lot of followership, the ability to have strong engagement in a culture of sharing was considered a criterion for leading and six participants alluded to this. For example, one participant mentioned: "In Quora there are a lot of people who would post [comment on a post] but won't write a post. The silent majority are probably people who read and don't interact at all"-P04. There was an agreement that most users on Quora were passive rather than active which necessitate that discussion leaders would strategize for a stronger participation. Moreover, user participation is vital to sustain the platform as a "third place" for $\mathrm{Q}$ and $\mathrm{A}$ where regular discussion leaders makes it lively without which it would be an empty space (Oldenberg, 1999).

The relationship between the leader and their following is strengthened by the focus topic or domain of interest and this was suggested as a reason for the differences in the success of influential people leading answers on Quora. According to the fifth participant: "Everybody is different on Quora with different perspectives. Some people are more successful than others and it depends on the topics they write about. Some topics I have written about got only about 500 views. After you've established yourself in some select topics that is more followed, you can broadcast your own message very effectively". The focus topic is usually what the participant cares about. Two participants agreed that taking up discussion leader roles on topics have attracted some attention; one of them stated: "I am diagnosed with Borderline Personality 
TABLE 3 | Coding interview data.

Interview extract/codes

"I would categorize being a leader as one who has lots of followership" -P01

"I have been influenced by post made on Quora" -P03

"I write and interact a lot but don't think of myself as a special leader" -P04

"Some people are more successful than others, it depends on topics they write" -P05

"You can accrue a following based on merit" -P06

"A person with a lot of followers on Quora" - P07

"I created my own page. When Quora introduced spaces, I was invited to be an admin for three pages" -P08

"People on Quora take a little bit more time to write answers unlike in Reddit" - P01

"I never seek it out." - P02

"I try to have well founded argument and express my bias" - P04

"After you've established yourself. . . You can broadcast your own message " -P05

"do something commercial do. . sell your product" -P06

"Start first by giving answers that people enjoy and getting their attention" -P07

"A post I made surprisingly ended out getting sent to about two million people" - P01

"I sometimes post funny answers to create humor for those reading my post" - P03

"I would not write answers that are inimical to societal values or on things" -P05

"I use copywriting tactics to make my answers. . there are topics I wouldn't lead" - P06

"First, you have to capture the audience's attention,.. with a controversial post" - P07

"I started writing to see if my understanding of my disorder was accurate" -P08

"One answer could be seen by a million people and another seen by five" -P01

"You have to respect your readers and not to give them an error-filled answers" -P02"

"Quora audience is more reflective of people on the internet and like Tiktok" -P06

"After you capture their attention, you keep it by writing interesting answers" -P07

"To just share what I knew for whoever would listen" -P01

"I do not want to gain anything. I enjoy answering questions" -P02

"no monetary motives.. To seek approval with up-votes and top-writer ranks" -P03

"I have a patreon (crowdfund) - I actually get paid by people to write on Quora" -P04

"For knowledge and sharing experience" -P05

"I hope to connect with other people on Quora" -P06

"To influence public perception about overlooked topics I care about" -P07

"To remove the stigma that is attached to my disorder" -P08

\section{Themes}

Leadership and followership relationship

Rethinking and inspiring followership

Mechanisms for eliciting interest

In praise of the admirer

Discerning motives
Disorder. I started writing to see if my own understanding of my disorder was accurate. I have no ability to perceive myself. It's part of the disorder. I have no identity. We can, without treatment, become emotional remoras. What I discovered was this. . to show what I write makes sense to many people. And my voice has a way of healing or helping others on their journey. I created my own page, Invisible Lines, mostly to blog my day to day frustrations. My own page did not take off, but when Quora introduced spaces, I was invited to be an admin for three pages."- P08. The following an individual has is able to determine their influence on Quora, and because the Ask-to-Answer design of the platform allows users to tag other Quora members, highly active users are promoted because they would be tagged in most questions increasing their influence (Paul et al., 2012). Five participants alluded to this and one particularly stated: "I try to influence public perception about topics I care about that are being overlooked and people don't have much information about it. I try to give my answers, make them popular so some people will learn about it for the first time and others who know about it will have complete information."-P07.

\section{Rethinking and Inspiring Followership}

The process of influencing followership and participation on Quora requires expertise and experience that builds up overtime. The fifth participant explicitly revealed that this building process is shrouded in mystery: "Sometimes you write answers that don't get any readership. I have noticed that if you focus on what you really know and what you can help people with, eventually your articles would start getting attention. This is very hard in the beginning. When I started on Quora and wrote an answer, I would be lucky if 100 or 200 people read it. Everybody that seems to be doing well on Quora in terms of readership, tend to write in the same subject and have a good broad knowledge and a certain message in the way they write." One way Quora can support newcomers sharing great ideas and leading discussions to increase their audience base could be by making their feeds visible in order to encourage other members to pay more attention to them (Burke et al., 2009).

Leadership theory is enriched by having an understanding of followership, and leaders have been reported to compare followers to their ideal expectation of followership, and judge them against this model sometimes without considering the actual behavior of the follower (Ford and Harding, 2018). Determining what scenarios would make a post trending on Quora, five participants agreed that this process takes time; P07 stated: "First, you have to capture the audience's attention, let's say If you have an intriguing picture or a controversial post or something like the corona virus. After you capture their attention, you have to keep it by writing answers that are interesting and easy to understand. Popularity of answers on Quora boils down to talking about things that are interesting where you have a unique perspective that keeps the audience's attention." While 
this may be true, some start with the audience in mind but rather posts what motivates their followership as posited by the fourth participant: "I don't worry about trending, but on writing good stuff and that is the reward. My post on coronavirus is just an example. Sharing answers on topics that have not been shared is a motivation. On Quora, I can trust the audience to choose what they want and don't want to absorb. I am very widely read and that is what makes my post trending."

\section{Mechanisms for Eliciting Interest}

People in active leadership roles could improve the relationship with followers by advancing connections that elevates, stimulates, and transform both followers and leaders into confident leaders and moral agents respectively (Burns, 1978). This perhaps could be the rationale why active leaders of discussion posts in online communities cherry-pick their topics to influence conversation. Answering the question on what will make a post trending on Quora the sixth participant stated: "I use copywriting tactics to make my answers. I post about sex usually on the first headline, because people are naturally gravitating towards that. Things like sex, racism, and something controversial generally elicits more comments. Although I would not like to do those things, sometimes when I have written answers of great value, they got almost no traction. Sometimes you have to do something commercials do such as the use of sex, cars to sell your product and then once they are inside you give better forms of advice." Another participant [P03] was also in agreement saying: "You want to make sure your answers are flawless and free of errors when you lead a discussion post ... when people see that your answers are impeccable, they would know you are competent and want to follow you." On the other hand, there were other contrasting views; participant one and two mentioned not seeking to influence followership, the first participant who was particularly surprised at the traction gotten by a recent post said: "I have been surprised a couple of times. There was a post I made that was a little bit technical and I did not imagine it would elicit much interest. It ended out getting sent to about two million people because they have an email where they broadcast messages to people, and it was a little bit surprising, I really don't know what causes them to be successful." An overview of the answers given showed that six participants alluded to the fact that what made an answer on Quora trending involved a strategic process and that if one is an expert in a range of topic and had a wealth of experience on advising, they would not be concerned with scenarios that makes a post trending. This agrees with the study by Meindl (1995) who stated that charisma is influenced by discussions among followers and it arises from the minds of admirers who concludes that a leader is charismatic.

\section{In Praise of the Admirer}

Emerging studies in leadership have been focused on followership that assumes that there is a common purpose for both leaders and their following, and a mutually beneficial relationship (Challef, 1998; Ciulla, 1998; Shamir et al., 2007). Those who lead discussions and write answers on Quora understand human behavior that allow effective followership to occur, and are self-aware of their audience. A participant [P03] on being asked for additional comment on experience writing answers on Quora mentioned: "Being a writer means that you are sometimes "naked" [revealing secrets] just in a bid to convince people that you are sure of what you are talking about. Many secrets about countries, government and people are being exposed in a bid to get up-votes." Apart from knowing their audience, they are also aware of the design of online communities and how they affect user behavior. A participant [P05] said: "In standard media you could get one answer, one article, one perspective unlike in Quora where you could get fifty articles if it's a popular question. Also, there are people on Quora who have not written anything but come there to get knowledge." Participant six also shared similar opinion: "I think one of the good things about Quora is that you could get answers to a question you really didn't know you needed an answer to. I think that the randomness of that makes the user experience more exciting, because for example, a big problem with Instagram which is a completely controlled platform [i.e. you are not seeing anything except things you want to see] therefore you are getting a very myopic sense of the world and there is a level of nonrandomness which gets boring and stale. The Quora stream is more similar to Tiktok where you get what you want. Those algorithms tend to heighten user experience."

Accordingly, there are similarities in the opinion of the participants that influential leaders of online discussion write answers using tactics that increases participation because they are aware of their audience, their content and the design of the online community. This self-awareness makes them reconsider feedback from their readers to keep the followership they have been able to acquire overtime. The eight-participant said: "For a while I used to write some trashy answers. I used to write answers with underlined italics to capture attention, but people were of the opinion that "this isn't cool" and that it looks like am hunting for attention and not providing as much value as I could, which made me rethink how am writing. Yes, I will change it if people point how badly am writing."

\section{Discerning Motives}

According to Mamykina et al. (2011) intrinsic rewards such as the desire for learning, altruism or extrinsic benefits such as social or material rewards have been highlighted as the motivations for participating in online question and answer sites. Some sites such as YouTube have a reward mechanism for active users with rich content, while other have point-based reward system (Mamykina et al., 2011). However, Quora is devoid of point-based reward system (Paul et al., 2012). To understand the intrinsic or extrinsic motivations for leading discussions, participants were asked what they hoped to gain from leading a discussion on Quora. Seven out of eight participants mentioned intrinsic rewards such as altruistic actions (helping people with advice, direction, spreading knowledge), social interaction and building social capital as the main motivations. Providing further information on the motives for writing answers on Quora [P03] stated: "Some people have come to Nigeria to meet me and learn more about the country and what I have written. Some of my followers who are in a dating relationship with a Nigerian call me to know about the country and verify the authenticity of the locations and its safety. Quora is a place where people build relationship and trust one 
another based on the answers and suggestions they are reading. It is one of the most visited sites in the world and I think people take post there seriously."

In addition, others have seized the networking opportunity provided by the online platform to create awareness to remove stigma attached to a disorder. P08 said: "My primary goal for writing on Quora is to remove the stigma that is attached to my disorder. Borderlines have a particularly brutal stigma attached to their disease. A whole lot of support are subjective and very few facts. Clinicians hate to treat us. We burn through people and their emotions like a woodchopper and it takes years of multidisciplined help to get anywhere near better. I have done a lot of work but still more to do. I also managed to create a community of both Borderlines, and former partners, who are willing to put aside their anger to get to a place of acceptance, radical acceptance. It feels very validating, that I have accomplished through real hard work, the ability to bridge the communication gap that is so frustrating about BPD." Furthermore, social appraisal is the popular reward mechanism that exists on Quora, and while some participants have leveraged on their popularity on Quora for extrinsic benefit from external sources, others have called on the designers of Quora to recognize and reward creative contributions. P06 said: "I think that Quora should add 'tip features' where people can give creators money. There is an immense amount of value being created on the internet, and the people that are creating it for the first time in human history are not getting access to profit from it. Am not saying that platforms should pay people, but, if someone writes an answer that changes your life [like benefits from YouTube] we can create a line between consumer of their content and the creators bank account. I think with this, we would get a better content; and this is the reason why YouTube has better videos than a lot of NetFlix documentaries."

The motivation to offer these services is aided by the design of Quora platform. It was suggested that the design algorithms on Quora frequently engineers related notifications to a user as unrelated feeds would discourage participation. On how an unrelated post would affect user experience the seventh participant stated: "I think Quora does a good job with its algorithms about giving you things you would be interested in. At some point I once interacted with a topic (conjoined twins) that I cared very little about but I enjoyed it and kept on getting questions and answers [scientific] about it and there's no way for me to indicate that am uninterested in it. And this causes more unrelated things to pop up in my feed and notification which drives me away from Quora."

\section{DISCUSSION/CONCLUSION}

Our study to understand the perspectives of those writing answers and leading discussions on Quora found that the motives for leading discussions were mostly intrinsic (altruism, knowledge sharing) than extrinsic (material reward) suggesting that providing balanced argument and reducing bias is highly valuable. The mechanisms that influences followership and participation in a discussion requires expertise and experience and this happens over time. It includes the ability to engage strongly and to improve relationship with followers by advancing connections that excites both parties (Burn, 1978). This greater knowledge of deliberative etiquette is needed to resist targeted incivility (Gervais, 2015). A well-thought-out answer was largely considered as a means of reaching a targeted audience and increasing participation, while biased and error-prone messages could lead to distrust. It can be suggested from the study that those who lead discussions and write answers on Quora are self-aware of their audience; and perhaps use tactics that increases participation. Copyright tactics, controversial answers and sharing new information were some of the highlighted mechanisms for influencing followership.

Exploring the concept of building leadership on the theory of followership, most participants were of the opinion that followership should not be a concern if one has the right message. However, it has been reported that leaders have an ideal expectation of followership that differs from reality (Ford and Harding, 2018). The study by Paul et al. (2012) to understand reputation mechanisms on Quora found that influential users get more votes than are deserving for their answers. This is further supported by Lerman and Galstyan (2008) who studying social voting on Digg noticed that some users on the site had their topics promoted in the front page of the platform because of their notoriety in large social networking sites. Although it has been reported that Quora uses an algorithm called PeopleRank to rank answers based on some select criteria, it has also been suggested that this could potentially lead to undue influence because it highly ranks the answers of popular users even when their answers are not catchy (Paul et al., 2012). This study suggests that active users influence follower perception, and the platform design of Quora promotes this influence by allowing users tag other members to a particular answer.

The small sample size of participants was a limitation of the study. Moreover, while thematic analysis offers flexibility and has the advantage of revealing the unknown unknowns, a drawback is the potential lack of coherence in developing the themes (Holloway and Todres, 2003). In this study, coherence was improved by using participant's explicit statements and position to support the themes created.

In summary, the main interest of the study was focused on mechanisms on how to gain followership by understanding leaders' actions and motivations to gain a following. This information is paramount in understanding the design implications for knowledge-building in online communities since the ability to create knowledge is important. Designers of online communities can leverage on this information, incorporating it into technology for building social support groups and geographically distributed communities in order to understand how knowledge is generated and disseminated by users, the changing status of interaction and knowledge in spaces dominated by key contributors, occasional contributors and newcomers, and the role a careful design of online community spaces play in providing added value in these environments. The C4P framework (content, conversation, connections, context and purpose) describes the elements that are vital for knowledge-building (Hoadley and Kilner, 2015). The C4P enables members of online communities to socialize, draw out meaningful knowledge from conversations, form collaborations and understand the context in which an information is conveyed with shared purpose (Hoadley and Kilner, 2015).

Knowing the active users and highly followed leaders would create conditions where they can be targeted by designers for specific 
contribution or even repackaging their content and conversation into new spaces. A participant [P08] acknowledged being invited be an admin for three pages on Quora when spaces were introduced, and this was after creating a page and dedicating it to helping people with borderline disorder. Understanding how knowledge spreads in online communities and the role technology plays potentially leads to a design framework that promotes learning through technology. While this study focused on understanding leader's motivation to gain a following, further studies may be carried out to investigate preferential attachments that influences social voting from the perspective of the follower. Also, understanding the way information is produced and circulated on Quora in relation to its ranking algorithm is another interesting area for future studies.

\section{DATA AVAILABILITY STATEMENT}

The raw data supporting the conclusions of this article will be made available by the authors, without undue reservation.

\section{REFERENCES}

Barabási, A.-L., and Albert, R. (1999). Emergence of scaling in random networks. Science 286 (5439), 509-512. doi:10.1126/science.286.5439.509

Bruckman, A. (2006). Teaching students to study online communities ethically. J. Inf. Ethics 3 (11), 82-95. doi:10.3172/jie.15.2.82

Burke, M., Lento, T., and Marlow, C. (2009). Feed me: motivating newcomer contribution in social network sites. Available at: http://www.thoughtcrumbs. com/publications/paper0778-burke.pdf (Accessed January 16, 2020).

Burns, J. (1978). Leadership. New York, NY: Harper and Row.

Carsten, M. K., Uhl-Bien, M., and Huang, L. (2018). Leader perceptions and motivation as outcomes of followership role orientation and behavior'. Leadership 14 (6), 731-756. doi:10.1177/1742715017720306

Challeff, I. (1998). The courageous follower: standing up to and for our leaders. San Francisco, CA: Berrett-Koehler Pub.

Ciulla, J. (1998). Ethics: the heart of leadership. London, ENG: Praeger.

Creswell, J. W. (2007). Qualitative inquiry and research design: choosing among five approaches: international student edition. Thousand Oaks, CA: Sage Publications.

D’Angelo, A. (2017). How many people use Quora. Available at: https://www. quora.com/How-many-people-use-Quora-3.

Evans, A. N., and Rooney, B. J. (2013). Methods in psychological research: chapter six: selecting research participants. 3rd edn. Edmonton, AB: Sage Publications, 440.

Ford, J., and Harding, N. (2018). Followers in leadership theory: fiction, fantasy and illusion. Leadership 14 (1), 3-24. doi:10.1177/1742715015621372

Gervais, B. T. (2015). Incivility online: affective behavioral reaction to uncivil political posts in a web-based experiment. J. Inf. Tech. Polit. 12 (2), 167-185. doi:10.1080/19331681.2014.997416

Guest, G., Namey, E., and Chen, M. (2020). A simple method to assess and report thematic saturation in qualitative research. PLoS One 15 (5), e0232076. doi:10. 1371/journal.pone.0232076

Hoadley, C. M., and Kilner, P. G. (2015). Using technology to transform communities of practice into knowledge-building communities, communities. Assoc. Comput. Machinery 25 (1). doi:10.1145/1067699.1067705

Holloway, I., and Todres, L. (2003). The status of method: flexibility, consistency and coherence. Qual. Res. 3357, 345. doi:10.1177/1468794103033004

Kim, A. (2003). Nine principles of community design. Available at: https://people. apache.org/ jim/NewArchitect/webtech/1998/01/kim/index.html (Accessed January 21, 2020).doi:10.1145/1119772.1119798

Kollock, P. (1998). Design principles for online communities. Harvard Conf. Internet Soc. 15 (5), 58-60.

Lerman, K., and Galstyan, A. (2008). “Analysis of social voting patterns on Digg," in WOSN'08: proceedings of the first workshop on online social networks (Seattle, WA: ACM Press), 7-12.

\section{ETHICS STATEMENT}

The study was reviewed and approved by University of Nebraska, IRB. The participants provided their informed consent to participate in this study.

\section{AUTHOR CONTRIBUTIONS}

MN developed the study question, designed the research, and typed the manuscript. CN contributed to the study concepts and design ideas.

\section{ACKNOWLEDGMENTS}

I would like to acknowledge Briana Morrison, Assistant Professor at University of Nebraska at Omaha for reviewing the study and supporting its publication.

Maity, S. K., Kharb, A., and Mukherjee, A. (2017). "Language use matters: analysis of the linguistic structure of question texts can characterize answerability in Quora," in Proceedings of the eleventh international AAAI conference on web and social media, Montreal, Quebec, Canada Available at : https://www.aaai.org/ocs/index.php/ICWSM/ICWSM17/ paper/view/15647/14866.

Mamykina, L., Manoim, B., Mittal, M., Hripcsak, G., and Hartmann, B. (2011). "Design lessons from the fastest Q and A site in the West," in Proceedings of the 2011 annual conference on human factors in computing systems (ACM Press), 2857-2866.

Meindl, J. R. (1995). The romance of leadership as a follower-centric theory: a social constructionist approach. The Leadership Quarterly 6, 329-341.

Nowell, L. S., Norris, J. M., White, D. E., and Moules, N. J. (2017). Thematic analysis: striving to meet the trustworthiness criteria, Int. J. Qual. Methods. doi:10.1177/1609406917733847

Oldenberg, R. (1999). The great good place. Available at: https://www.mnsSu.edu/ voices/greatgoodplace.pdf (Accessed March 3, 2020).

Patil, S., and Lee, K. (2016). Detecting experts on Quora: by their activity, quality of answers, linguistic characteristics and temporal behaviors. Soc. Netw. Anal. Min. 6, 5. doi:10.1007/s13278-015-0313-x

Paul, S. A., Hong, L., and Chi, E. H. (2012). Who is authoritative? Understanding reputation mechanisms in quora. Available at: https://www.researchgate.net/ publication/224053714 (Accessed March 3, 2020).

Peh, J. (2017). How many questions have been asked on Quora. Available at: https://www.quora.com/How-many-questions-have-been-asked-on-Quora-1/ answers/19190364 (Accessed March 3, 2020).

Quora (2020). Why quora exist. Available at: https://www.quora.com/about (Accessed February 26, 2020).

Shamir, B., Pillai, R., and Bligh, M. (2007). Follower-centered perspectives on leadership: a tribute to the memory of James R. Greenwich, CT: Information Age Publishing.

Strauss, A., and Corbin, J. (1990). Basics of qualitative research: grounded theory procedures and techniques. Newbury Park, CA: Sage Publications.

Tolstikov-Mastand Yulia (2016). Global followership: the launch of the scholarly journey. Adv. Glob. Leadership 2016, 107-150. doi:10.1108/ S1535-120320160000009013

Vollstedt, M., and Rezat, S. (2019). An introduction to grounded theory with a special focus on axial coding and the coding paradigm. Compend. Early Career Res. Math. Edu. 13, 81-100. doi:10.1007/978-3-030-15636-7_4

Wang, G., Gill, K., Mohanlal, M., Zheng, H., and Zhao, B. Y. (2013). Wisdom in the social crowd: an analysis of Quora," in WWW'13: proceedings of the 22nd international conference on World WideWeb (Rio de Janeiro, Brazil: ACM) 
Warwick Institute for Employment Research (IER) (2014). Analyzing interview data. University of Warwick. Available at: https://warwick.ac. $\mathrm{uk} / \mathrm{fac} / \mathrm{cross}$ fac/esrcdtc/researchandtraining/ct201314/quals/analysing interview_data_2014_wk3_for_web.pdf (Accessed March 23, 2020).

Weeks, B. E., Ardèvol-Abreu, A., and de Zúñiga, G. H. (2017). Online influence? Social media use opinion leadership persuasion. Int. J. Public Opin. Res. 29 (2), 214-239. doi:10.1093/ijpor/edv050

Zhu, H., Kraut, R., and Kittur, A. (2012). "Effectiveness of shared leadership in online communities," in CSCW'12 proceedings Of the ACM conference on computer supported cooperative work, 407-416.
Conflict of Interest: The authors declare that the research was conducted in the absence of any commercial or financial relationships that could be construed as a potential conflict of interest.

Copyright (c) 2021 Nwadiugwu and Nwadiugwu. This is an open-access article distributed under the terms of the Creative Commons Attribution License (CC BY). The use, distribution or reproduction in other forums is permitted, provided the original author(s) and the copyright owner(s) are credited and that the original publication in this journal is cited, in accordance with accepted academic practice. No use, distribution or reproduction is permitted which does not comply with these terms. 